\title{
Multiple Goal Linear Programming-Based Decision Preference Inconsistency Recognition and Adjustment Strategies
}

\author{
Jian-Zhang $\mathrm{Wu}^{*}$ (D), Li Huang, Rui-Jie $\mathrm{Xi}$ and Yi-Ping Zhou \\ School of Business, Ningbo University, Ningbo 315211, China \\ * Correspondence: sjzwjz@gmail.com
}

Received: 25 April 2019; Accepted: 27 June 2019; Published: 1 July 2019

\begin{abstract}
The purpose of this paper is to enrich the decision preference information inconsistency check and adjustment method in the context of capacity-based multiple criteria decision making. We first show that almost all the preference information of a decision maker can be represented as a collection of linear constraints. By introducing the positive and negative deviations, we construct the the multiple goal linear programming (MGLP)-based inconsistency recognition model to find out the redundant and contradicting constraints. Then, based on the redundancy and contradiction degrees, we propose three types of adjustment strategies and accordingly adopt some explicit and implicit indices w.r.t. the capacity to test the implementation effect of the adjustment strategy. The empirical analyses verify that all the strategies are competent in the adjustment task, and the second strategy usually costs relatively less effort. It is shown that the MGLP-based inconsistency recognition and adjustment method needs less background knowledge and is applicable for dealing with some complicated decision preference information.
\end{abstract}

Keywords: decision preference information; capacity; inconsistency check and adjustment; multiple goal linear programming; volume of feasible range

\section{Introduction}

In the context of multiple criteria decision making, the probability measure or the weight vector mainly reflects the importance relationship of decision criteria and assumes all the criteria are absolutely independent of each other. Replacing the additivity of the probability measure by the monotonicity w.r.t. set inclusion, capacity [1], or the fuzzy measure [2], we obtain the powerful ability to construct a variety of nonadditivity situations to represent the complex interaction phenomena among decision criteria [3-6]. One of the most critical tasks for the capacity-based decision making pattern is to represent the decision maker's preference information efficiently and consistently.

The efficient approaches to represent the decision maker's preference information usually include the following three categories. The first category of approaches is to show the decision maker's general preference by a particular type of capacity, which is suitable for some specific decision situation and meanwhile has a relatively smaller amount of coefficients than the ordinary capacity. For example, the $p$-symmetric capacity means that the decision criteria consist of $p$ classes of indifferent criteria $[7,8]$; the $k$-additive capacity takes into account the interaction among at most $k$ criteria and has a simple structure [3,9]; the $k$-tolerant and $k$-intolerant capacity respectively express that any $k$ criterion is enough to favor or vote for an alternative [10]; the $k$-maxitive and -minitive capacities respectively focus on the lower and upper $k$-order subsets' capacity values concerning only the micro and macro view of decision criteria [5,11-13]. The second category of approaches utilizes kinds of equivalent representations of capacity. Different representations have different decision meanings. 
For example, the Shapley importance and interaction index shows the overall importance of criteria and comprehensively the simultaneous interaction among them [3,14,15]; Möbius representation represents the internal simultaneous interaction of criteria [16]; the nonadditivity index describes the internal interaction associated with nonadditivity [17]. The third category of approaches is to adopt the fuzzy integral [18], most commonly the Choquet integral [1], to show directly the decision maker's preference for some typical alternatives, which can be carried out by the desired overall evaluations or the partial order on alternatives [19-21]. These three approaches provide a systematic scheme to show comprehensively the decision preference information, and most of them can be established in terms of linear constraints.

Almost all the decision preference information represented in terms of these three categories can be transformed into linear constraints about one or more entries, like criteria, alternatives, or kinds of indices about criteria or alternatives. Because of the complexity and uncertainty of the decision making problem, as well as the variety of the preference presentation approaches mentioned above, it is inevitable that some contradictory and redundant information exists among the collection of decision maker's preferences. These inconsistencies can lead to some unnecessary burdens on the model's construction and computation, and even an incompatible situation. Hence, the inconsistencies' recognition and adjustment method and strategy becomes a vital task for the capacity-based decision pattern.

The inconsistency check and adjustment approaches of this correlative preference information can be classified into two major branches. One branch uses the 0-1 mixed linear programming to find out the contradicting constraints and then directly removes them from the set without any further programmable process of adjustment [22-24]. Another branch uses multiple goal linear programming (MGLP) to recognize the contradictory, as well as the redundant constraints and then provides some adjustment suggestions [5].

In the paper, we will focus on the second branch and enhance the MGLP model with programmable steps and detailed adjustment strategies. The advantage of this enhanced method is that it (a) does not need much prior knowledge of the decision preference, but (b) can figure out the inconsistency degrees of all constraints simultaneously even when they are established in quite different kinds of indices or representations, and then (c) can flexibly customize the adjustment measures according to the contradictory and redundancy degrees of the inconsistent constraints. Some empirical cases for these programmable steps and adjustment strategies are also given.

Another task of this paper is to propose some measurements to quantify the effects of different adjustment strategies. By the adjustment process, an infeasible constraints collection becomes a feasible region of compatible capacities. How to compare explicitly the effects of different adjustment strategies turns out to be a practical research topic. In this paper, we adopt some indices, like the orness (andness) of capacity [4], the Shapley interaction index of the empty set, and the Choquet integral values of typical alternatives, to show the volume of a given feasible range of capacity. One main advantage of these selected indicators to show the adjustment performance is that they are all linear combinations of capacity values, other than the nonlinear indicators, such as the entropy index [25], or some quadratic distance to the uniform capacity, and then, some modeling and calculating conveniences by using the linear programming technologies and software packages can be achieved accordingly.

This paper is organized as follows. After the Introduction, we present some knowledge about the capacity, its representations, particular types of capacities, and the Choquet integral in Section 2. In Section 3, we summarize the linear constraint representation form of the decision maker's preference information. Section 4 is devoted to the MGLP-based inconsistency checking algorithm and adjustment strategies. We give some adjustment performance evaluation indices and verify the real effects of the adjustment strategies through a few empirical examples in Section 5. Finally, we conclude the paper in Section 6. 


\section{Preliminaries}

Let $N=\{1,2, \ldots, n\}, n \geq 2$, be the set of decision criteria, $\mathcal{P}(N)$ be the power set of $N$, and $|S|$ be the cardinality of subset $S \subseteq N$. The definitions listed in this section can be found in $[1-3,6,7,10,14,16-18,20,26-29]$.

Definition 1. A set function $\mu: \mathcal{P}(N) \rightarrow[0,1]$ is called a capacity on $N$ if: (i) $\mu(\varnothing)=0, \mu(N)=1$; (ii) $\forall A, B \subseteq N, A \subseteq B$ implies $\mu(A) \leq \mu(B)$.

The value of $\mu(A)$ directly reflects the decision maker's perception of the importance of criteria in $A$ to the decision problem.

Capacity has many other equivalent representation forms, i.e., the one-to-one linear mappings, most of which have special decision meanings and explanations.

Definition 2. Let $\mu$ be a capacity on $N$. The Möbius representation of $\mu$ is defined as:

$$
m_{\mu}(A)=\sum_{B \subseteq A}(-1)^{|A \backslash B|} \mu(B), A \subseteq N
$$

Möbius representation is usually considered as the internal simultaneous interaction of the criteria coalition. Actually, it has another vital role in capacity-based decision making theory: it is very efficient at presenting capacity and the Choquet integral, as well as at constructing capacity identification methods and, hence, successfully provides another systematic representation approach of the capacity-related theory.

Definition 3. Let $\mu$ be a capacity on $N$. The Shapley importance and interaction index of $\mu$ is defined as:

$$
I_{\mu}(A)=\sum_{B \subseteq N \backslash A} \frac{1}{(|N|-|A|+1)}\left(\begin{array}{c}
|N|-|A| \\
|B|
\end{array}\right)^{-1}\left(\sum_{C \subseteq A}(-1)^{|A \backslash C|} \mu(C \cup B)\right), A \subseteq N
$$

Theoretically, the Shapley importance and interaction index extends the notion of the Shapley value, which is a core concept in multiple criteria decision making and the cooperative game theory. In the above equation, if the $A=\{i\}$, the index $I_{\mu}(\{i\})$, or $I_{\mu}(i)$ for convention, is just the Shapley value of criterion $i$. It can be explained as the probabilistic expectation of the marginal contributions of the criterion to all other coalitions of criteria and is generally taken to scale the overall importance of this criterion for the decision problem. When $A$ is nonempty and non-singleton, the index $I_{\mu}(A)$ can be explained as the probabilistic expectation of the marginal interaction of criteria in $A$ with the presence of all other subsets and is generally taken to measure the comprehensively simultaneous interaction of criteria in $A$.

Definition 4. The nonadditivity index of subset $A \subseteq N$ w.r.t. $\mu$ is defined as:

$$
n_{\mu}(A)=\mu(A)-\frac{1}{2^{|A|-1}-1} \sum_{C \subset A} \mu(C) .
$$

By using the nonadditivity index, the decision maker can represent the internal interaction of the criteria coalition associated with nonadditivity. This index is constructed based on the common sense that strict superadditive (resp. strict subadditive, superadditive, and subadditive) capacity means decision criteria are mutually complementary (substitutive, non-substitutive, and non-complementary).

The decision maker's whole preference information on decision criteria can alternatively be represented by adopting a special type of capacity. 
Definition 5. Let $k \in\{1,2, \ldots, n\}$. A capacity $\mu$ is said to be $k$-additive if its Möbius representation satisfies $m_{\mu}(A)=0$ for all $A \subseteq N$ such that $|A|>k$ and there exists at least one subset $A$ of $k$ elements such that $m_{\mu}(A) \neq 0$.

From this definition, one can figure out that $k$-additive capacity only takes into account the internal simultaneous interaction; accordingly, the comprehensive simultaneous interaction, among at most $k$ criteria, ignores higher order internal and comprehensive interactions.

Definition 6. A capacity $\mu$ on $N$ is said to be p-symmetric if the coarsest partition of $N$ into subsets of indifference contains exactly $p$ subsets $A_{1}, \ldots, A_{p}$, where $A_{i}$ is a subset of indifference, $A_{i} \cap A_{j}=\varnothing$, $\cup_{i=1}^{p} A_{i}=N, i, j=1, \ldots, p$, and a partition $\pi$ is coarser than another partition $\pi^{\prime}$ if all subsets of $\pi$ are the union of some subsets of $\pi^{\prime}$. The partition $\left\{A_{1}, \ldots, A_{p}\right\}$ is called the basis of $\mu$.

One-symmetric capacity is just the symmetric capacity or the symmetric weight vector. In each indifference subset $A_{i}$, the decision criteria are totally indifferent, they have the same importance and interaction characteristics in the decision process. The $p$-symmetric capacity is suitable for the decision making with $p$ groups of anonymous appraisal experts or indiscriminating points of views.

Definition 7. Let $k \in\{1, \ldots, n\}$. A capacity $\mu$ on $N$ is said to be $k$-tolerant if $\mu(A)=1$ for all $A \subseteq N$ such that $|A| \geq k$ and there exists a subset $B \subseteq N$, with $|B|=k-1$, such that $\mu(B) \neq 1$. A capacity $\mu$ on $N$ is said to be $k$-intolerant if $\mu(A)=0$ for all $A \subseteq N$ such that $|A| \leq n-k$ and there exists a subset $B \subseteq N$, with $|B|=n-k+1$, such that $\mu(B) \neq 0$.

The $k$-tolerant capacity describes a relative tolerant attitude for the decision alternative, in which the alternative can be regarded as excellent as long as it satisfies arbitrary $k$ criteria not necessarily more that even all of the $n$ criteria. On the contrary, the $k$-intolerant capacity constructs an intolerant decision situation that an alternative will be totally denied only if it fails on arbitrary $k$ criteria at most.

Definition 8. Let $k \in\{1, \ldots, n\}$. A capacity $\mu$ on $N$ is said to be $k$-maxitive if $\mu(A)=\bigvee_{B \subset A,|B| \leq k} \mu(B)$ for $|A| \geq k+1$ and there exists a subset $C \subseteq N$, with $|C|=k$, such that $\mu(C) \neq \bigvee_{B \subset A} \mu(B)$. A capacity $\mu$ on $N$ is said to be $k$-minitive if $\mu(A)=\bigwedge_{B \subset A,|B| \geq n-k} \mu(B)$ for $|A| \geq n-k-1$ and there exists a subset $C \subseteq N$, with $|C|=n-k$, such that $\mu(C) \neq \bigwedge_{B \subset A} \mu(B)$.

The $k$-maxitive and -minitive capacities respectively generalize the $k$-tolerant and $k$-intolerant capacities and can present the decision maker's preference or judgment from the micro and macro perspective. That is, the $k$-maxitive (minitive) capacity only needs to identify the lower (upper) $k$-order capacity values and then just use the max ( $\mathrm{min}$ ) operation to generate the other subsets' capacity values accordingly.

The Choquet integral is a widely-accepted form of fuzzy integral to aggregate flexibly the partial evaluation information of decision alternatives on multiple interdependent criteria.

Definition 9. Let $x$ be a real-valued function on $N, x:=\left(x_{1}, \ldots, x_{n}\right) \in[0,1]^{n}$. The Choquet integral of $x$ w.r.t. a capacity $\mu$ on $N$ is defined as $C_{\mu}(x)=\sum_{i=1}^{n}\left[x_{(i)}-x_{(i-1)}\right] \mu\left(N_{(i)}\right)$ or equally by $C_{\mu}(x)=\sum_{i=1}^{n}\left[\mu\left(N_{(i)}\right)-\mu\left(N_{(i+1)}\right)\right] x_{(i)}$ where the parentheses used for indices represent a permutation on $N$ such that $x_{(1)} \leq \cdots \leq x_{(n)}, x_{(0)}=0, N_{(i)}=\{(i), \ldots,(n)\}$, and $N_{(n+1)}=\varnothing$.

The Choquet integral can be accordingly used to present the decision maker's preference on the alternatives, which is basically the implicit form of preference on the decision criteria. 


\section{Linear Constraints Represent Decision Preference Information}

In general, the decision maker's preference information on criteria or alternatives shown in terms of the notions mentioned in Section 2 can be mathematically transformed into linear constraints.

From Definitions 2-4, we can get that Möbius representation, Shapley importance, interaction index, and nonadditivity index are all the linear combination of capacity values. From Definition 9, we can see that, if the partial evaluations of the decision alternative is given, the Choquet integral is obviously a linear combination of capacity values. Furthermore, the decision maker's preference information of these notions is usually provided by comparison or interval form $[5,9,19,22,24,30]$, like:

- the overall importance of criterion $i$ is at least as great as that of criterion $j: I_{\mu}(\{i\}) \geq I_{\mu}(\{j\})$;

- the sign of the internal simultaneous interaction of criteria subset $A$ is positive or negative: $m_{\mu}(\{i, j\}) \geq(\leq) 0$;

- $\quad$ the comprehensive simultaneous interaction of criteria subset $A$ is at least as great as that of $B$ : $I_{\mu}(A) \geq I_{\mu}(B)$;

- the intensity of the nonadditivity index of criteria subset $A$ is at least as great as that of $B$ : $\left|n_{\mu}(A)\right| \geq\left|n_{\mu}(B)\right| ;$

- $\quad$ the nonadditivity index of criteria subset $A$ belongs to interval $[0.1,0.5]: 0.1 \leq n_{\mu}(A) \leq 0.5$;

- alternative $\mathbf{a}$ is at least as $\operatorname{good}$ as $\mathbf{b}: \mathcal{C}(\mathbf{a}) \geq \mathcal{C}(\mathbf{b})$;

- $\quad$ alternative $\mathbf{a}$ is as good as $\mathbf{b}:|\mathcal{C}(\mathbf{a})-\mathcal{C}(\mathbf{b})| \leq \delta$, where $\delta$ is a small threshold, e.g., 0.05 .

The modulus in the above fourth constraint can be removed if the signs of index values are given. The last constraint can be directly transformed as two linear constraints: $\mathcal{C}(\mathbf{a})-\mathcal{C}(\mathbf{b}) \leq \delta$ and $\mathcal{C}(\mathbf{a})-\mathcal{C}(\mathbf{b}) \geq-\delta$. Hence, all the above constraints can be formulated as linear constraints of capacities, the equivalent linear representations of capacity, and the fuzzy integrals.

As for the special types of capacities, we can also transform them into a collection of linear constraints. It is easy to represent $k$-additive capacity as linear constraints since the Möbius representation is just a linear combination of capacity; see Definitions 2 and 5 . For the $p$-symmetric capacity with basis $\left\{A_{1}, \ldots, A_{p}\right\}$, we can construct a $p$-dimensional vector $\mathbf{b}_{S}=\left(b_{1}, \ldots, b_{p}\right), S \subseteq N$, where $b_{i}=\left|S \cap A_{i}\right|, i=1, \ldots, p$, then the $2^{n}$ coefficients of capacity can be transformed into $\prod_{i=1}^{p}\left(\left|A_{i}\right|+1\right)$ coefficients of $\mathbf{b}_{S}$ and are still the linear combinations of capacity basically. It is also very easy to represent the $k$-tolerant and -intolerant capacity since it just sets some of the capacity values as zero or one, obviously linear constraints. For the $k$-maxitive and minitive capacities, we can construct the following linear constraint sets as [5]:

$$
\begin{gathered}
k \text {-maxitive }\left\{\begin{array}{l}
\mu(A)-\mu(A \backslash\{i\}) \leq y_{A, i},|A| \geq k+1, i \in A, \\
y_{A, i}=\left\{\begin{array}{l}
0 \text { if } \mu(A)-\mu(A \backslash\{i\})=0 \\
1 \text { if } \mu(A)-\mu(A \backslash\{i\}) \neq 0
\end{array}\right. \\
\sum_{A} y_{A, i} \leq|A|-1 .
\end{array}\right. \\
k \text {-minitive }\left\{\begin{array}{l}
\mu(A \cup\{i\})-\mu(A) \leq y_{A, i},|A| \leq n-k-1, i \in N \backslash A, \\
y_{A, i}=\left\{\begin{array}{l}
0 \text { if } \mu(A \cup\{i\})-\mu(A)=0 \\
1 \text { if } \mu(A \cup\{i\})-\mu(A) \neq 0
\end{array}\right. \\
\sum_{A} y_{A, i} \leq n-|A|-1 .
\end{array}\right.
\end{gathered}
$$

With these linear constraints, if they are compatible reciprocally, we can obtain a feasible range of available capacities. Based on this, the most satisfactory capacity can be obtained by introducing the learning set or explicit preference dependent principles; see, e.g., [5,19,20,24,25,30-38]. If the linear constraints are incompatible or inconsistent, we can adopt the following algorithm to recognize the inconsistency and adjust them to the consistent case by some strategies. 


\section{MGLP-Based Inconsistency Recognition and Adjustment Algorithm}

In this section, we discuss the algorithm and strategy of recognizing and adjusting the inconsistency by using MGLP. The algorithm helps decision makers to firstly recognize the inconsistent and redundant constraints according to the deviation degrees, and further provide some suggestions and strategies to adjust the inconsistent and redundant constraints.

\subsection{MGLP Model and Inconsistency Degree}

For convenience, we first transform all the directions of linear constraints into a uniform type of "equal to and greater than", $\geq$. That is, if the direction of constraints is opposite, we just multiply both hand sides by -1 . Then, we can construct the preference information-based MGLP model by introducing the positive and negative deviation variables, $d_{r}^{+}$and $d_{r}^{-}$, where $r=1, \ldots, p$ and $p$ is the total amount of all the preference constraints, to each preference constraint. These obtained goal linear constraints are all equations. For example, constraint $\mathcal{C}(\mathbf{a}) \geq \mathcal{C}(\mathbf{b})$ will become the goal constraint as:

$$
\mathcal{C}(\mathbf{a})-\mathcal{C}(\mathbf{b})-d_{r}^{+}+d_{r}^{-}=0 .
$$

The objective function of the MGLP is just the sum of all the positive and negative deviations. That is, the MGLP model can be established as:

$$
\min \sum_{r=1}^{p} d_{r}^{+}+d_{r}^{-}
$$

s.t. all the goal constraints of decision preference information,

the boundary and monotonicity of ordinary capacity,

the linear constraints of a special type of capacity (optional),

where $d_{r}^{+}, d_{r}^{-} \geq 0$.

Then, by solving the MGLP model, we get the optimal solutions, in which the optimal deviations are just the inconsistency degrees of the preference constraints. Accordingly, the constraints can be classified into three categories. The first are the consistent constraints with both optimal deviation variables equal to zero, $d_{r}^{+*}=d_{r}^{-*}=0$. Other constraints with optimal positive or negative deviation not being zero, $d_{r}^{+*} \neq 0$ or $d_{r}^{-*} \neq 0$ and $d_{r}^{+*} \neq d_{r}^{-*}$, can be considered as the inconsistent constraints. In more detail, the constraints with $d_{r}^{-*} \neq 0$ are called the contradictory constraints (the second category), and the constraints with $d_{r}^{+*} \neq 0$ are called the redundancy constraints (the third category). The deviation variable $d_{r}^{-*}$ can be considered as the contradiction degree of the preference constraint, $d_{r}^{+*}$ the redundancy degree, and $\max \left\{d_{r}^{+*}, d_{r}^{-*}\right\}$ the inconsistency degree.

\subsection{Three Types of Adjustment Strategies}

One can easily figure out that the infeasibility of model 1 is caused by the contradictory constraints other than the redundant constraints. Therefore, it is better to keep the redundant constraints other than removing or revising them because they are helpful for keeping the diversity of preference information, as well as the volume of the feasible range of compatible capacity. On the contrary, the contradictory constraints must be adjusted to obtain a nonempty feasible range of capacity. The adjustment can be carried out by the following three strategies:

S1 Remove all the contradictory constraints;

S2 Adjust all the contradictory constraints simultaneously;

S3 Iterate to adjust the most contradictory constraint till we obtain a consistent case for all constraints.

Strategy $\mathrm{S} 1$ is the most radical adjustment strategy. Although it will undoubtedly get the largest compatible collection of preference constraints, it is obvious that too much preference information has been directly omitted and abandoned. Strategy S2 also provides an efficient approach to obtain the 
consistent situation of preference information with moderate adjustment effort in these three strategies. Strategy S3 undertakes an iterative adjustment process with more efforts compared to the other two strategies, which also means more interactive participation from the decision maker can be employed in Strategy S3.

For a given contradictory constraint, besides removing it from the preference set, two types of further adjustment measures can be adopted. One is to just subtract the contradiction degrees $d_{r}^{-*}$ on the right-hand side of the constraint, which is applicable for any type of preference (the comparison or interval form). This measure is very suitable to deal with many constraints simultaneously, like the situation of Strategy S2, to get a wholly consistent case. The other is to reverse the direction of the constraint, e.g., to change $\mathcal{C}(\mathbf{a}) \geq \mathcal{C}(\mathbf{b})$ into $\mathcal{C}(\mathbf{a}) \leq \mathcal{C}(\mathbf{b})$. This measure leads to the contrary preference of the initial one, so it needs to be adopted carefully and is not suitable to apply simultaneously to many constraints in general.

\section{Adjustment Effect Appraisal Indices and Empirical Analysis}

\subsection{Volume of the Feasible Range of Capacity}

The effect of the adjustment strategy can be appraised through the volume of the feasible range of capacity. Generally, if the feasible range is the empty set, i.e., the preference constraints have some inconsistencies, then its volume can be regarded as zero naturally. However, when feasible range is nonempty, it is usually infinite and hard to tell its scale exactly. Here, we introduce some indices and use their range (difference between maximum and minimum) to reflect the volume of the feasible range indirectly.

The first index is the entropy of a capacity $\mu$ on $N$, which is defined by [25]:

$$
E(\mu)=\sum_{i=1}^{n} \sum_{A \subseteq N \backslash\{i\}} \frac{(n-|A|-1) !|A| !}{n !} h(\mu(A \cup\{i\})-\mu(A)),
$$

where $h(x)=-x \ln x$ if $x>0$ and zero if $x=0$. The symmetric additive capacity has the largest entropy value $\ln (n)$. The Choquet integral w.r.t. such a measure coincides with the arithmetic mean function. The entropy value can be considered as a kind of similarity measure with symmetric additive capacity.

The second index is the orness index of capacity, which is defined as [10]:

$$
\operatorname{orness}(\mu)=\sum_{A \subset N} \frac{(n-|A|) !|A| !}{n !(n-1)} \mu(A) .
$$

The orness basically describes the aggregation trend of the Choquet integral w.r.t. the capacity, i.e., it behaves more like a max or min operator.

The third index can be the Shapley index value of the empty set (see Definition 3),

$$
I_{\mu}(\varnothing)=\sum_{A \subseteq N} \frac{1}{(|N|+1)}\left(\begin{array}{l}
|N| \\
|A|
\end{array}\right)^{-1} \mu(A) .
$$

The Shapley index value of the empty set is also called the expectation of the Choquet integral $[10,30]$ and basically has a similar indication effect as the orness index, as the latter is just a kind of normalization form of the former.

The fourth index can be the Choquet integral of a given alternative, which can be generated randomly or constructed deliberately. The partial evaluations of this alternative on decision criteria are easier to differentiate from each other, e.g., alternative $(1 / n, 2 / n, \ldots, 1)$ could be a competent candidate in most situations.

The ranges of the above four indices can serve as the indicators to reflect the volume of a feasible range of capacity. Except the entropy index, the other three indices are all linear combinations of 
capacity values and have been chosen in the following examples because of the convenience of constructing and solving the optimization models by using mathematical computation packages, like the "lpSolve" package in the R language.

\subsection{Empirical Analysis of Some Cases}

Suppose there are six decision alternatives, $A_{1}, \ldots, A_{6}$, and five decision criteria, $N=\{1,2,3,4,5\}$. The partial evaluations of the alternatives on the five criteria are given in Table 1 . It should be mentioned that there are numerous practical decision cases with multiple criteria from the point of view of an enterprise or a regional government; see, e.g., [39-47]. Here, for the convenience of explanation, we focus on this empirical example.

Table 1. The partial evaluations of alternatives.

\begin{tabular}{cccccc}
\hline Alternative & Criterion 1 & Criterion 2 & Criterion 3 & Criterion 4 & Criterion 5 \\
\hline$A_{1}$ & 0.228 & 0.586 & 0.973 & 0.113 & 0.395 \\
$A_{2}$ & 0.706 & 0.387 & 0.551 & 0.656 & 0.346 \\
$A_{3}$ & 0.147 & 0.400 & 0.664 & 0.326 & 0.387 \\
$A_{4}$ & 0.842 & 0.701 & 0.708 & 0.688 & 0.807 \\
$A_{5}$ & 0.886 & 0.692 & 0.877 & 0.820 & 0.360 \\
$A_{6}$ & 0.200 & 0.400 & 0.600 & 0.800 & 1.000 \\
\hline
\end{tabular}

Let us first run a primary test for the MGLP model to show its steps. We only give one constraint:

$$
\mathcal{C}\left(A_{1}\right) \geq \mathcal{C}\left(A_{1}\right)+0.1
$$

which is obviously an infeasible constraint. Transform the constraint into the goal constraint: $\mathcal{C}\left(A_{1}\right)-\mathcal{C}\left(A_{1}\right)-d^{+}+d^{-}=0.1$. According to Model (1), we get the following MGLP:

$$
\begin{aligned}
& \quad \min d^{+}+d^{-} \\
& \text {s.t. } \\
& \mathcal{C}\left(A_{1}\right)-\mathcal{C}\left(A_{1}\right)-d^{+}+d^{-}=0.1, \\
& \text { the boundary and monotonicity of ordinary capacity on } N,
\end{aligned}
$$

where $d^{+}, d^{-} \geq 0$. Solving the model, we can get $d^{-*}=0.1$, which means the constraint is not feasible. Then, we need to change the initial constraint as:

$$
\mathcal{C}\left(A_{1}\right) \geq \mathcal{C}\left(A_{1}\right)+0.1-d^{-*}, \text { i.e., } \mathcal{C}\left(A_{1}\right) \geq \mathcal{C}\left(A_{1}\right),
$$

which is a feasible constraint (actually no decision meaning).

In the following, we mainly investigate the adjustment strategies.

(I) The Shapley interaction index represents preference.

The decision maker believes that:

- the comprehensive interactions of $\{1,2\},\{1,3\},\{1,4\},\{1,5\}$, and $\{3,5\}$ are positive, at least greater than 0.05 ;

- the comprehensive interactions of $\{2,3\},\{2,4\},\{2,5\},\{3,4\}$, and $\{4,5\}$ are negative, at least smaller than -0.05 ;

- From a comprehensive view, Criterion 1 is more important than 2; 2 more is important than 4; 4 is more important than $5 ; 5$ is more important than 3; 3 is more important than 2 (all these constraints have a threshold of 0.1);

- the comprehensive interactions of $\{1,2,3\}$ are greater than those of $\{2,3,4\}$; the comprehensive interactions of $\{2,3,4\}$ are greater than those of $\{1,2,4\}$; the comprehensive interactions of $\{1,2,4\}$ are greater than those of $\{2,4,5\}$; the comprehensive interactions of $\{2,4,5\}$ are greater 
than those of $\{3,4,5\}$; the comprehensive interactions of $\{3,4,5\}$ are greater than those of $\{2,3,4\}$ (all these preference constraints have a threshold of 0.1).

Accordingly, these preference can be transformed into the following linear constraints of the Shapley interaction indices, labeled as $\mathbf{c 1} \sim \mathbf{c 2 0}$ :

$$
\begin{gathered}
\text { c1: } I_{\mu}(\{1,2\}) \geq 0.05, \\
\quad \cdots \\
\text { c6: } I_{\mu}(\{2,3\}) \geq 0.05, \\
\quad \cdots \\
\text { c11: } I_{\mu}(\{1\}) \geq I_{\mu}(\{2\})+0.1, \\
\quad \cdots \\
\text { c16: } I_{\mu}(\{1,2,3\}) \geq I_{\mu}(\{2,3,4\})+0.1,
\end{gathered}
$$

By constructing and solving the MGLP model, we can have:

$d_{12}^{-*}=0.35467703, d_{15}^{-*}=0.04532297$ and $d_{17}^{-*}=0.4$.

By examining the preference constraints, one can find that the first two inconsistency degrees are caused by the preference of the comprehensive importance of criteria:

$$
\begin{aligned}
& \text { c12: } I_{\mu}(\{2\}) \geq I_{\mu}(\{4\})+0.1 ; \\
& \text { c13: } I_{\mu}(\{4\}) \geq I_{\mu}(\{5\})+0.1 ; \\
& \text { c14: } I_{\mu}(\{5\}) \geq I_{\mu}(\{3\})+0.1 ; \\
& \text { c15: } I_{\mu}(\{3\}) \geq I_{\mu}(\{2\})+0.1 .
\end{aligned}
$$

Obviously, this is an inconsistent dominance loop. The latter contradiction degree is caused by another preference loop:

$$
\begin{aligned}
& \text { c17: } I_{\mu}(\{2,3,4\}) \geq I_{\mu}(\{1,2,4\})+0.1 ; \\
& \text { c18: } I_{\mu}(\{1,2,4\}) \geq I_{\mu}(\{2,4,5\})+0.1 ; \\
& \text { c19: } I_{\mu}(\{2,4,5\}) \geq I_{\mu}(\{3,4,5\})+0.1 ; \\
& \text { c20: } I_{\mu}(\{3,4,5\}) \geq I_{\mu}(\{2,3,4\})+0.1 .
\end{aligned}
$$

Now, the three adjustment strategies are applied to the preference to achieve the consistency cases. The constraints $\mathbf{c 1 2}, \mathbf{c 1 5}$, and $\mathbf{c 1 7}$ are removed if we use S1. By adopting S2, the three contradiction degrees are subtracted from their corresponding preference constraints. For the S3, we adjust the contradictory constraints by sequentially subtracting $0.4,0.36666667$, and 0.03333333 from the right-hand sides of the constraints $\mathbf{c 1 7}, \mathbf{c 1 2}$ and c15. One can see that S3 is slightly different from S2 in the adjustment amounts on the three constraints. The adjustment effects of the three strategies are given in Table 2, in the row "c1 $\sim \mathbf{c 2 0}$ ". One can see that $\mathrm{S} 1$ has the maximum ranges on three effect indices; S2 is better than S3 on almost every effect index. Taking into account the effort of adjusting and the presentation of initial preference information, S2 is a good choice.

(II) The nonadditivity index represents preference.

Different from the Shapley interaction index, the nonadditivity has a uniform range $[-1,1]$ for all subsets even with different cardinalities. Therefore, we can just divide $[-1,1]$ into eight equivalent segments: [ $-1,-0.75],[-0.75,-0.5],[-0.5,-0.25],[-0.25,0],[0,0.25],[0.25,0.5],[0.5,0.75]$, and $[0.75,1]$ to represent the extremely negative, strongly negative, negative, slightly negative, slightly positive, positive, strongly positive, and extremely positive nonadditive index (the interaction kind and degree associated with nonadditivity). The decision maker believes that: 
- the nonadditivity indices of $\{1,2,3\},\{1,2,4\},\{2,3,4\},\{1,2,3,4\}$, and $\{2,3,4,5\}$ are extremely positive, strongly positive, strongly positive, negative, and strongly negative, respectively.

Each preference of the nonadditivity index will become two constraints, e.g., for $\{1,2,3\}$, there are $n_{\mu}\{1,2,3\} \geq 0.75$ and $n_{\mu}\{1,2,3\} \leq 1$ (or equivalently, $-n_{\mu}\{1,2,3\} \geq-1$ ). We labeled the constraint of nonadditivity indices as $\mathbf{c 2 1} \sim \mathbf{c 3 0}$. By constructing and solving the MGLP model, we can have:

$d_{28}^{-*}=0.2142857$ and $d_{30}^{-*}=0.1428571$.

In the optimal solution of the MGLP model, a few pieces of redundant information can be figured out. For example, $d_{29}^{+*}=0.3928571$, which equals $d_{30}^{-*}+0.25$, means that $\mathbf{c 2 9}$ is a redundant constraint. However, as mentioned in Section 4, it is better to keep this constraint, as it gives a meaningful constraint in the decision, $n_{\mu}\{2,3,4,5\} \geq-0.75$. The adjustment effects of three strategies are also given in Table 2, in the row "c21 c $3 \mathbf{3 0}^{\prime}$, where S2 is the same as S3.

(III) The preference information on alternatives.

The decision maker believes that:

- $\quad A_{1}$ is better than $A_{5}$;

- $A_{2}$ is better than $A_{4}$;

- $A_{2}$ is better than $A_{5}$;

- $\quad A_{3}$ is better than $A_{4}$;

- $\quad A_{3}$ is better than $A_{6}$.

All five constraints have a threshold of 0.1 , labeled as c31 c 35. By constructing and solving the MGLP model, we can have:

$d_{31}^{-*}=0.04, d_{32}^{-*}=0.152, d_{33}^{-*}=0.321, d_{34}^{-*}=0.144$ and $d_{35}^{-*}=0.036$.

This means all the constraints are contradictory. Adjustment Strategy S1 removes all these constraints. We apply Strategy S2 by subtracting the contradiction degrees from the right-hand sides of the five constraints. For S3, we iterate to adjust the model according to the following rule: if the maximum contradiction degree is larger than the threshold 0.1 , then reverse the corresponding constraint; else subtract the maximum contradiction degree from its right-hand side. The adjustment result of S3 is: subtract 0.01189421 for c31; subtract 0.04342418 for c35; and reverse the other three constraints. The adjustment effects of three strategies are still given in Table 2; see the row "c31 c 35 ".

(IV) The composition of all the above preference information.

If we collect all the above preference information into one MGLP model and solve it, we can get the following contradiction degrees:

$$
\begin{aligned}
& d_{1}^{-*}=0.033653846, d_{2}^{-*}=0.032692308, d_{8}^{-*}=0.133653846, d_{11}^{-*}=0.133653846, \\
& d_{12}^{-*}=0.308012821, d_{13}^{-*}=0.068269231, d_{14}^{-*}=0.023717949, d_{20}^{-*}=0.4 \\
& d_{28}^{-*}=0.293681319, d_{30}^{-*}=0.401923077, d_{31}^{-*}=0.513758333, d_{32}^{-*}=0.251000641, \\
& d_{33}^{-*}=0.292763462, d_{34}^{-*}=0.488621154, d_{35}^{-*}=0.468687821
\end{aligned}
$$

Adjustment Strategy S1 still removes all the above inconsistent conditions associated with the above contradiction degrees. For S2, we execute the following two measures: the first is to subtract all the contradiction degrees from the right-hand sides of the inconsistent constraints, denoted as S2-1; the second is to remove the contradictory constraints with the contradiction degree larger than 0.1 and subtract the right-hand sides of the contradictory constraints by their contradiction degrees, denoted as S2-2. Strategy S3 iteratively adjusts the most contradictory condition by using the following rule: reverse the constraint if its contradiction degree is greater than 0.1 , else just subtract the contradiction degree. The adjustment effects of these four strategies can be found in row "c1 $\sim \mathbf{c} 35$ " of Table 2 . It should be pointed out that Strategy S2-1 actually obtains a singular capacity; all the ranges' upper boundaries are equal to their lower boundaries. This situation usually occurs as a compromise of a great many contradictory constrains. 
Table 2. The adjustment effects of the three strategies.

\begin{tabular}{lllll}
\hline $\begin{array}{l}\text { Preference } \\
\text { Constraints }\end{array}$ & $\begin{array}{l}\text { Adjustment } \\
\text { Strategies }\end{array}$ & Orness Index & $\begin{array}{l}\text { Shapley Index of the } \\
\text { Empty Set }\end{array}$ & $\begin{array}{l}\text { Choquet Integral of } \\
A_{\mathbf{6}}\end{array}$ \\
\hline \multirow{3}{*}{$\mathbf{c 1} \sim \mathbf{c 2 0}$} & $\mathrm{S} 1$ & {$[0.2677954,0.6370333]$} & {$[0.3451969,0.5913555]$} & {$[0.2313801,0.7633333]$} \\
& S2 & {$[0.3079380,0.6366931]$} & {$[0.3719587,0.5911287]$} & {$[0.3784653,0.7359502]$} \\
& S3 & {$[0.3250942,0.6346277]$} & {$[0.3833961,0.5897518]$} & {$[0.4359961,0.7384137]$} \\
\hline \multirow{3}{*}{$\mathbf{c 2 1} \sim \mathbf{c 3 0} 0$} & S1 & {$[0.16875,0.7375]$} & {$[0.2791667,0.6583333]$} & {$[0.3,1]$} \\
& S2 & {$[0.7312144,0.7375]$} & {$[0.6541429,0.6583333]$} & {$[0.99994,1]$} \\
& S3 & {$[0.7312144,0.7375]$} & {$[0.6541429,0.6583333]$} & {$[0.99994,1]$} \\
\hline \multirow{3}{*}{$\mathbf{c} 31 \sim \mathbf{c 3 5}$} & S1 & {$[0,1]$} & {$[0.1666667,0.8333333]$} & {$[0.2,1]$} \\
& S2 & {$[0.625,0.8]$} & {$[0.5833333,0.7]$} & {$[0.6,0.6]$} \\
& S3 & {$[0.5013613,0.8716408]$} & {$[0.5009075,0.7477605]$} & {$[0.5792869,0.6074242]$} \\
\hline \multirow{4}{*}{$\mathbf{c 1} \sim \mathbf{c 3 5}$} & S1 & {$[0.375,0.6678002]$} & {$[0.4166667,0.6118668]$} & {$[0.3961406,0.8620681]$} \\
& S2-1 & {$[0.6010417,0.6010417]$} & {$[0.5673611,0.5673611]$} & {$[0.7447436,0.7447436]$} \\
& S2-2 & {$[0.5302842,0.6010417]$} & {$[0.5201895,0.5854089]$} & {$[0.6281133,0.6396871]$} \\
& S3 & {$[0.4357052,0.5502848]$} & {$[0.4571368,0.5335232]$} & {$[0.5244241,0.6829989]$} \\
\hline
\end{tabular}

From Table 2, one can see that Strategy S1 always has the maximum ranges of the three indices, which means the largest volume of compatible capacities. As mentioned in Section 4, S1 obtains the consistent case by just removing all contradictory constraints, and too much useful preference information is directly abandoned. To contrast, Strategies S2 and S3 can reserve the decision maker's preference information to a large extent. Furthermore, S2 and S3 have similar effects in most situations, especially when they employ a similar detailed adjustment measure. For these two strategies, S2 is more efficient and has less adjustment effort, compared to S3. Therefore, in general, S2 can be the most desirable strategy to adjust the inconsistent constraints.

\section{Conclusions}

In this paper, we discussed and presented the linear constraint representation of the decision maker's preference information in terms of capacity, its equivalent transformations, the special types, as well as the Choquet integral. By using these linear constraints, the decision maker can flexibly and comprehensively represent his/her explicit and implicit preference on decision alternatives and decision criteria. We elaborated the steps of the MGLP-based inconsistency recognition method and proposed three main adjustment strategies. The empirical study showed that all three strategies are practicable and that Strategy S2 can be the first choice in most situations.

It was also verified that the implementation of the inconsistency recognition and some of the adjustment methods, e.g., directly subtracting inconsistency degrees from the right-hand sides, does not need much field knowledge of the decision problem. The proposed method can also deal with the relatively complicated preference constraints with the mixed representations of multiple indices of capacities and nonlinear integrals. Furthermore, some flexible adjustment strategies can also be adopted and applied if there is more available decision context information. Absolutely, the intimate cooperation and participation of decision makers in the adjustment process is very vital for a high quality decision, and the decision aid package of executing the proposed methodology is also helpful in practice.

Finally, we want to mention that in the proposed method of this paper, all the preference constraints were treated equally, i.e., the coefficients of all deviations in the objective function were totally the same. Actually, these coefficients can be used to show the decision maker's preemptive or weighted priorities on the preference constraints. The effects of the scale and ratio of these coefficients on the optimal capacities also is a meaningful topic for future research. The software package for executing the proposed method and strategies is also very helpful for decision making in practice. 
Author Contributions: Conceptualization, J.-Z.W.; methodology, J.-Z.W.; software, L.H.; validation, R.-J.X. and Y.-P.Z.; data curation, R.-J.X.; writing—original draft preparation, J.-Z.W. and L.H.; writing-review and editing, J.-Z.W. and L.H.; project administration, J.-Z.W.

Funding: The work was supported by the National Natural Science Foundation of China (No. 71671096) and the K.C. Wong Magna Fund of Ningbo University.

Conflicts of Interest: The authors declare no conflict of interest.

\section{References}

1. Choquet, G. Theory of capacities. Ann. De L'inst. Fourier 1954, 5, 131-295. [CrossRef]

2. Sugeno, M. Theory of Fuzzy Integrals and Its Applications. Ph.D. Thesis, Tokyo Institute of Technology, Tokyo, Japan, 1974.

3. Grabisch, M. k-order additive discrete fuzzy measures and their representation. Fuzzy Sets Syst. 1997, 92, 167-189. [CrossRef]

4. Grabisch, M. Set Functions, Games and Capacities in Decision Making; Springer: Berlin, Germany; New York, NY, USA, 2016.

5. Beliakov, G.; James, S.; Wu, J.Z. Discrete Fuzzy Measures: Computational Aspects; Springer: Cham, Switzerland, 2019.

6. Wu, J.Z.; Beliakov, G. Probabilistic bipartition interaction index of multiple decision criteria associated with the nonadditivity of fuzzy measures. Int. J. Intell. Syst. 2019, 34, 247-270. [CrossRef]

7. Miranda, P.; Grabisch, M.; Gil, P. p-Symmetric fuzzy measures. Int. J. Uncertain. Fuzziness Knowl.-Based Syst. 2002, 10, 105-123. [CrossRef]

8. Wu, J.Z.; Yu, L.P.; Li, G.; Jin, J.; Du, B. The sum interaction indices of some particular families of monotone measures. J. Intell. Fuzzy Syst. 2016, 31, 1447-1457. [CrossRef]

9. Wu, J.Z.; Zhang, Q. 2-order additive fuzzy measure identification method based on diamond pairwise comparison and maximum entropy principle. Fuzzy Optim. Decis. Mak. 2010, 9, 435-453. [CrossRef]

10. Marichal, J.L. k-intolerant capacities and Choquet integrals. Eur. J. Oper. Res. 2007, 177, $1453-1468$. [CrossRef]

11. Mesiar, R. k-order additive fuzzy measures. Int. J. Uncertain. Fuzziness Knowl.-Based Syst. 1999, 7, 561-568. [CrossRef]

12. Wu, J.Z.; Beliakov, G. Nonadditive robust ordinal regression with nonadditivity index and multiple goal linear programming. Int. J. Intell. Syst. 2019, 34, 1732-1752. [CrossRef]

13. Beliakov, G.; Wu, J.Z. Learning fuzzy measures from data: Simplifications and optimisation strategies. Inf. Sci. 2019, 494, 100-113. [CrossRef]

14. Shapley, L.S. A value for n-person games. Contrib. Theory Games 1953, 2, 307-317.

15. Fujimoto, K.; Kojadinovic, I.; Marichal, J.L. Axiomatic characterizations of probabilistic and cardinal-probabilistic interaction indices. Games Econ. Behav. 2006, 55, 72-99. [CrossRef]

16. Chateauneuf, A.; Jaffray, J.Y. Some characterizations of lower probabilities and other monotone capacities through the use of Möbius inversion. Math. Soc. Sci. 1989, 17, 263-283. [CrossRef]

17. Wu, J.Z.; Beliakov, G. Nonadditivity index and capacity identification method in the context of multicriteria decision making. Inf. Sci. 2018, 467, 398-406. [CrossRef]

18. Wang, Z.; Klir, G.J. Generalized Measure Theory; Springer: New York, NY, USA, 2010.

19. Grabisch, M.; Kojadinovic, I.; Meyer, P. A review of methods for capacity identification in Choquet integral based multi-attribute utility theory: Applications of the Kappalab R package. Eur. J. Oper. Res. 2008, 186, 766-785. [CrossRef]

20. Grabisch, M.; Labreuche, C. A decade of application of the Choquet and Sugeno integrals in multi-criteria decision aid. Ann. Oper. Res. 2010, 175, 247-286. [CrossRef]

21. Wu, J.Z.; Zhou, Y.P.; Huang, L.; Dong, J.J. Multicriteria correlation preference information (MCCPI) based ordinary capacity identification method. Mathematics 2019, 7, 300. [CrossRef]

22. Angilella, S.; Greco, S.; Matarazzo, B. Non-additive robust ordinal regression: A multiple criteria decision model based on the Choquet integral. Eur. J. Oper. Res. 2010, 201, 277-288. [CrossRef]

23. Corrente, S.; Greco, S.; Kadziński, M.; Słowiński, R. Robust ordinal regression in preference learning and ranking. Mach. Learn. 2013, 93, 381-422. [CrossRef] 
24. Greco, S.; Mousseau, V.; Slowinski, R. Ordinal regression revisited: Multiple criteria ranking using a set of additive value functions. Eur. J. Oper. Res. 2008, 191, 416-436. [CrossRef]

25. Marichal, J.L. Entropy of discrete Choquet capacities. Eur. J. Oper. Res. 2002, 137, 612-624. [CrossRef]

26. Weber, R.J. Probabilistic values for games. In The Shapley Value. Essays in Honor of Lloyd S. Shapley; Roth, A., Ed.; Cambridge University Press: Cambridge, UK, 1988; pp. 101-119.

27. Banzhaf, J.F., III. Weighted voting doesn't work: A mathematical analysis. Rutgers L. Rev. 1964, $19,317$.

28. Beliakov, G.; Sola, H.B.; Calvo, T. A Practical Guide to Averaging Functions; Springer: New York, NY, USA, 2016.

29. Wu, J.Z.; Beliakov, G. Nonmodularity index for capacity identifying with multiple criteria preference information. Inf. Sci. 2019, 492, 164-180. [CrossRef]

30. Wu, J.Z.; Pap, E.; Szakal, A. Two kinds of explicit preference information oriented capacity identification methods in the context of multicriteria decision analysis. Int. Trans. Oper. Res. 2018, 25, 807-830. [CrossRef]

31. Meyer, P.; Roubens, M. Choice, Ranking and Sorting in Fuzzy Multiple Criteria Decision Aid. In Multiple Criteria Decision Analysis: State of the Art Surveys; Figueira, J., Greco, S., Ehrogott, M., Eds.; Springer: New York, NY, USA, 2005; pp. 471-503.

32. Wu, J.Z.; Zhang, Q.; Du, Q.; Dong, Z. Compromise principle based methods of identifying capacities in the framework of multicriteria decision analysis. Fuzzy Sets Syst. 2014, 246, 91-106. [CrossRef]

33. Marichal, J.L.; Roubens, M. Determination of weights of interacting criteria from a reference set. Eur. J. Oper. Res. 2000, 124, 641-650. [CrossRef]

34. Beliakov, G. Construction of aggregation functions from data using linear programming. Fuzzy Sets Syst. 2009, 160, 65-75. [CrossRef]

35. Kojadinovic, I. Minimum variance capacity identification. Eur. J. Oper. Res. 2007, 177, 498-514. [CrossRef]

36. Kojadinovic, I. An axiomatic approach to the measurement of the amount of interaction among criteria or players. Fuzzy Sets Syst. 2005, 152, 417-435. [CrossRef]

37. Wu, J.Z.; Yang, S.; Zhang, Q.; Ding, S. 2-additive capacity identification methods from multicriteria correlation preference information. IEEE Trans. Fuzzy Syst. 2015, 23, 2094-2106. [CrossRef]

38. Corrente, S.; Greco, S.; Ishizaka, A. Combining analytical hierarchy process and Choquet integral within non-additive robust ordinal regression. Omega 2016, 61, 2-18. [CrossRef]

39. Lee, S.H. Applying system dynamics to strategic decision making in construction. Front. Eng. Manag. 2017, 4, 35-40. [CrossRef]

40. Kazimieras Zavadskas, E.; Antucheviciene, J.; Chatterjee, P. Multiple-Criteria Decision-Making (MCDM) Techniques for Business Processes Information Management. Information 2018, 10, 4. [CrossRef]

41. Ziemba, P. Towards Strong Sustainability Management-A Generalized PROSA Method. Sustainability 2019, 11, 1555. [CrossRef]

42. Stojčić, M.; Zavadskas, E.K.; Pamučar, D.; Stević, Ž.; Mardani, A. Application of MCDM Methods in Sustainability Engineering: A Literature Review 2008-2018. Symmetry 2019, 11, 350. [CrossRef]

43. López-Morales, V. Multiple Criteria Decision-Making in Heterogeneous Groups of Management Experts. Information 2018, 9, 300. [CrossRef]

44. Stojić, G.; Stević, Ž.; Antuchevičienè, J.; Pamučar, D.; Vasiljević, M. A Novel Rough WASPAS Approach for Supplier Selection in a Company Manufacturing PVC Carpentry Products. Information 2018, 9, 121. [CrossRef]

45. Ziemba, P. Inter-Criteria Dependencies-Based Decision Support in the Sustainable wind Energy Management. Energies 2019, 12, 749. [CrossRef]

46. Siksnelyte, I.; Zavadskas, E.K.; Streimikiene, D.; Sharma, D. An Overview of Multi-Criteria Decision-Making Methods in Dealing with Sustainable Energy Development Issues. Energies 2018, 11, 2754. [CrossRef]

47. Adeel, A.; Akram, M.; Ahmed, I.; Nazar, K. Novel m-Polar Fuzzy Linguistic ELECTRE-I Method for Group Decision-Making. Symmetry 2019, 11, 471. [CrossRef]

(C) 2019 by the authors. Licensee MDPI, Basel, Switzerland. This article is an open access article distributed under the terms and conditions of the Creative Commons Attribution (CC BY) license (http:/ / creativecommons.org/licenses/by/4.0/). 